\title{
Multiple Sensory Neuronal Correlates of Site-Specific Sensitization in Aplysia
}

\author{
Edgar T. Walters \\ Department of Physiology and Cell Biology, University of Texas Medical School at Houston, Houston, Texas 77225
}

\begin{abstract}
Noxious stimulation of a restricted site on the skin of Aplysia (training) causes site-specific sensitization of the tailwithdrawal reflex that is associated with several sensory correlates that are evident both $10 \mathrm{~min}$ and $2 \mathrm{hr}$ after training. First, extracellularly recorded afferent activity evoked by test stimulation of the trained site increases, indicating peripheral sensory changes. Second, central sensory alterations are manifested by tail sensory neurons within the pleural VC cluster that innervate the trained site and are activated during training. These mechanosensory/nociceptive cells display a number of differences from unactivated tail sensory neurons innervating other sites: (1) slow depolarization of the soma observed immediately after training, (2) decrease in soma action potential threshold, and (3) enhancement of monosynaptic EPSPs to identified motor neurons. Noxious stimulation of a more extensive region also produces site-specific sensitization of the tail-withdrawal reflex and site-specific enhancement of EPSP amplitude measured $1 \mathrm{~d}$ after training. This training produced a novel cellular correlate of behavioral enhancement in Aplysia-regenerative bursting responses (2-35 spikes) in response to brief depolarization of the sensory nouron soma. The changes in peripheral and central excitability appear similar to changes associated with mammalian models of primary hyperalgesia. Site-specific enhancement of nociceptive signaling also occurs during aversive associative conditioning in a noxious unconditioned stimulus (US) pathway. These site-specific changes involve activity-dependent extrinsic modulation (ADEM) of the VC sensory neurons, suggesting a close relationship to changes underlying associative conditioning in conditioned stimulus (CS) pathways in Aplysia.
\end{abstract}

The preceding paper (Walters, 1987) showed that strong or injurious cutaneous stimulation produces site-specific sensitization of defensive reflexes in Aplysia that can last for a week. It was suggested that these changes are the functional equivalent of long-term "hyperalgesia" described in mammalian models (e.g., Woolf, 1984) and that site-specific sensitization may have

\footnotetext{
Received Mar. 10, 1986; revised July 21, 1986; accepted July 22, 1986.
}

This work was supported by National Institutes of Mental Health Grant MH38726, National Institutes of Health Research Career Development Award NS00848, and by the Chicago Community Trust/Searle Scholars Program. I thank L. Foster for technical assistance and Dr. J. H. Byrne for comments on an earlier draft of this paper.

Correspondence should be addressed to Dr. Edgar T. Walters, Department of Physiology and Cell Biology, University of Texas Medical School at Houston, P.O. Box 20708, Houston, TX 77225.

Copyright (C) 1987 Society for Neuroscience $0270-6474 / 87 / 020408-10 \$ 02.00 / 0$ been a link in the evolution of some forms of classical conditioning from general sensitization. In order to explore the mechanisms of site-specific sensitization and its possible relationships to "hyperalgesia" and to other forms of memory, it is important to examine its underlying neuronal processes. In particular, if neural correlates of "hyperalgesia" in Aplysia are similar to those observed in mammals, "hyperalgesia" in this simple preparation becomes an attractive model for investigating potentially general cellular mechanisms of short- and long-term nociceptive information processing. One mechanism that has been suggested for site-specific reflex enhancement by noxious stimulation is activity-dependent extrinsic modulation (ADEM) of sensory neurons. Although there is evidence for the involvement of ADEM in some forms of associative conditioning (Hawkins et al., 1983; Walters and Byrne, 1983a, b), its involvement in site-specific sensitization has only been implicated indirectly, by experiments at the behavioral level (Walters, 1987).

This paper describes neural correlates of site-specific sensitization in Aplysia displaying several interesting similarities to neural correlates of nociceptive sensitization and primary hyperalgesia in mammalian preparations. In addition, the central cellular correlates are consistent with the involvement of ADEM of nociceptive sensory neurons as a mechanism of site-specific sensitization. These correlates include a regenerative bursting response in the sensory neurons that has not previously been linked to behavioral changes. Finally, evidence is provided that site-specific sensitization occurs in an unconditioned stimulus (US) pathway during associative conditioning. Some of these results have appearcd in abstract form (Walters, 1985).

\section{Materials and Methods}

Studies were performed on Aplysia californica (200-400 gm) supplied by Alacrity Marine Biological Services (Redondo Beach, CA). Animals were maintained in artificial seawater ("Instant Ocean") at $19^{\circ} \mathrm{C}$ and kept at a relatively constant body weight with small daily meals of romaine lettuce prior to the experiments. In all experiments a reduced semi-intact preparation of the tail and surgically desheathed pedal-pleural ganglia (Walters et al., 1983a) was used for testing neuronal correlates of training. Training was conducted either in the semi-intact preparation or in the intact animal the day before testing. Dissections were carried out after anesthetizing the animal with injections of isotonic $\mathrm{MgCl}_{2}$ (equal to about $50 \%$ of the animal's weight)

Short- and intermediate-term correlates: Training in the semi-intact preparation. In these experiments (Figs. 2-8, 11) only the left pedalpleural ganglia were connected to the tail, by nerve Lp9. When behavioral responses were measured, they were recorded simultaneously with electrophysiological activity (both extra- and intracellular). Tail withdrawal was measured with a Grass FT.03 tension transducer, while extracellular activity was monitored with a suction electrode attached to a Grass P511J AC preamplifier (Fig. 1). The extracellular data were processed with a Modular Instruments (Southwestern, PA) acquisition system controlled by a Compaq Deskpro microcomputer. Afferent ac- 
tivity in ncrve Lp9 was sclcctively monitorcd during reversible blockade of axonal conduction between the suction electrode and the ganglia; filling the well (Fig. 1) with Na-free, isotonic $\mathrm{MgCl}_{2}$ solution prevented efferent spikes from reaching the electrode. Intracellular recordings of tail sensory and motor neurons were made using conventional techniques (Walters et al., 1983a). Only sensory neurons that had baseline EPSPs between 1 and $12 \mathrm{mV}$ were accepted (except for the study represented in Fig. 11). Larger EPSPs often show less facilitation, presumably because of a ceiling effect, and the lack of precision in measuring very small baseline EPSPs would contribute extra variability to the normalized scores.

Test and training stimuli were delivered to the dorsal surface of the left half of the tail through implanted silver wires insulated except for about $3 \mathrm{~mm}$ at the tip. Test stimuli were either single or double DC pulses (5-10 msec), the intensity of which was adjusted to causc a very weak, but repeatable, tail withdrawal when applied at $10 \mathrm{~min}$ intervals. Tests were conducted alternately with $\mathrm{Lp} 9$ blocked by $\mathrm{MgCl}_{2}$ and with conduction reinstated with artificial seawater. One of the 2 test sites was randomly selected to receive the brief training sequence. In alternate experiments the timing of the training relative to the conduction status of the nerve was reversed: In half, the training occurred following a "blocked" test, and in the other half the training occurred following a "normal" test. The actual training always occurred while conduction was normal between the ganglia and tail. The training stimulus consisted of ten $0.5 \mathrm{sec}$ trains at $5 \mathrm{~min}$ intervals. The amplitude of the pulses during testing and training was the same, but during training approximately 100 pulses were delivered in 10 high-frequency $(25 \mathrm{~Hz})$ bursts. This cutaneous training sequence was nearly identical to that applied to the nerve to produce long-term cnhancement of sensory neuron connections in the isolated ganglia (Walters and Byrne, 1985) and very similar to that used to produce long-term enhancement of reflexes in the intact animal (Walters, 1987).

Intracellular test stimuli were delivered to the somata of 2 tail sensory neurons every $10 \mathrm{~min}$. Each test consisted of a rapid, ascending series of successively larger depolarizing pulses ( $10 \mathrm{msec}$ each) that continued until an action potential was triggered. One sensory neuron's receptive field included the cutaneous electrode used for training; thus, this sensory neuron was activated by both the intracellular stimuli and by the test and training stimuli applied through the training electrode in the tail. Although both sensory neurons were located in the tail region of the VC cluster (Walters et al., 1983a), in these experiments only the "trained" cell was activated by cutaneous stimuli. Because the receptive ficlds of the sensory neurons are small, it can take a long time to find the receptive field of a given cell. In addition, the repeated cutaneous stimulation used to hunt for receptive fields can itself cause unwanted sensitization. Therefore, each experiment was begun as soon as 2 sensory neurons were found with monosynaptic connections to a tail motor neuron, with 1 of the sensory neurons being activated by 1 of the cutaneous test stimuli. Since the other "control" sensory neuron was also in the tail region of the VC cluster (and produced a monosynaptic EPSP in the motor neuron), it can be assumed to innervate another site on the tail separate from the training site (Walters et al., 1983a). To be an adequate control for the effects of training, it was important to show that the extra action potential(s) fired during each test in the trained cell (due to activation by the cutaneous test pulse) did not itself cause synaptic facilitation. Evidence for the adequacy of this control is presented in the Results.

Long-term correlates: Training in the intact animal. In a separate set of experiments (Figs. 9, 10) the training was applied to the intact animal the day before electrophysiological testing was conducted in the semiintact preparation. A spatially extensive training procedure was used, designed to activate as many sensory neurons as possible in the ipsilateral pleural VC cluster (Walters et al., 1983a). Approximately fifty $0.5 \mathrm{sec}$ trains $(60 \mathrm{~Hz} \mathrm{AC})$ of strong shock $(60 \mathrm{~mA})$ were applied at 2 sec intervals through bipolar capillary electrodes to the dorsal surface of one half of the animal, from the posterior part of the neck back along the ipsilateral parapodium and body wall to the tip of the tail. To minimize chances of activating sensory neurons in the contralateral pleural ganglion, shocks were not delivered close to the midline of the tail or the neck. The following morning the intact animal was tested behaviorally with weak $10 \mathrm{~mA}$ shocks $(0.5 \mathrm{sec})$ applied to each side of the tail, separated by $15 \mathrm{~min}$ (see Walters, 1987).

Shortly after the second behavioral test the animal was anesthetized and dissected, and the bilateral pairs of pedal-pleural ganglia were surgically desheathed in order to test the VC sensory neurons (Walters et

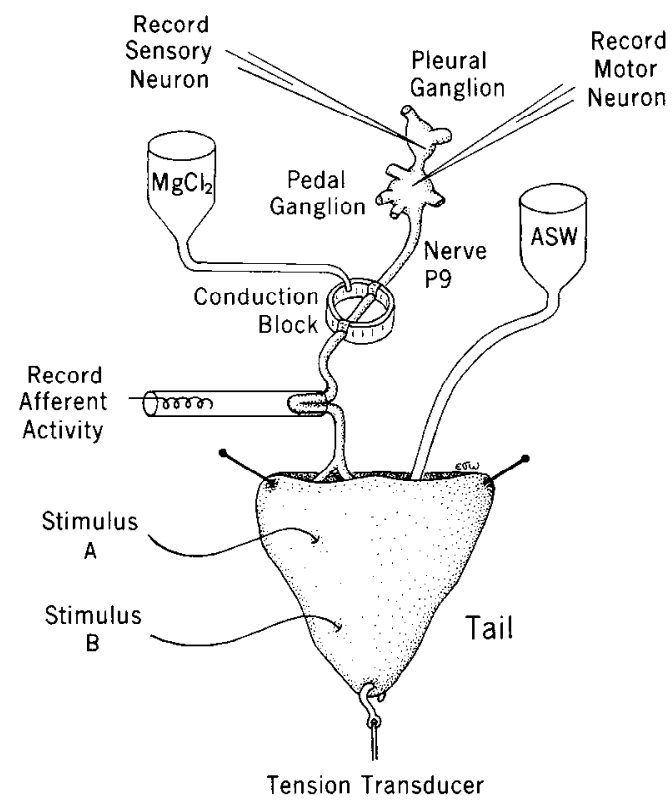

Figure 1. Semi-intact tail preparation. In some cases additional restraining pins were inserted along the right side of the tail. The tail was constantly perfused with aerated artificial seawater $(A S W)$. Test and training stimuli were applied through insulated silver electrodes implanted at sites $A$ and $B$. The resulting tail withdrawals were monitored with a tension transducer. Only the left pedal-pleural ganglia were left attached to the tail (by nerve Lp9), which could be functionally disconnected by reversibly blocking axonal conduction in the well with isotonic $\mathrm{MgCl}_{2}$. With the nerve blocked, afferent activity in the distal part of the nerve was recorded en passant with a suction electrode. The pedalpleural ganglia were surgically desheathed, allowing intracellular recording from tail motor neurons in the pedal ganglion and from various sensory neurons in the pleural VC cluster.

al., 1983a). The person conducting the electrophysiological tests did not know which side of the animal had been trained. Prior to testing, a scarch of cach pedal ganglion was made for homologous tail motor neurons, identified by position, electrophysiological characteristics, and, most importantly, similarity in movements produced in the tail following intracellular activation (Walters et al., 1983a). A coin-flip was then used to select the side to begin testing, and the following testing protocol was applied to each side. Twenty sensory neurons were sampled sequentially along the medial margin of each VC cluster, beginning with the cell at the posterior-medial corner of the cluster, continuing along the medial margin, going around the next (anterior-medial) corner, and finally ending about half-way along the medial margin in the anterior part of the cluster. Previous knowledge of the somatotopic mapping of these clusters (Walters et al., 1983a) allowed the inference that the sampled cells innervate the ipsilateral side of the tail and the ipsilateral parapodial region. Each sensory neuron was tested with a series of successively larger $10 \mathrm{msec}$ depolarizations produced by current injection into the cell body through the recording electrode. Once action potential threshold was reached, the microelectrode was withdrawn and inserted into the next sensory neuron. The entire testing sequence took 15-30 min on each side.

Statistical analysis. Unless otherwise stated, 2 a priori hypotheses based upon preliminary pilot studies were tested: (1) that the trained site would elicit larger test responses than the untrained site shortly after training (10-20 min test) and (2) that a memory of training would be expressed by the enhancement of the trained site responses relative to the control site responses when tested in either the "intermediate-term" $(110-120 \mathrm{~min}$ test) or "long-term" (1 d test). One-tailed $t$ tests for correlated means were used to test differences that had been predicted by pilot experiments, and the central tendency was represented by the mean $( \pm$ SEM). In each case the response distributions wcre plotted and in all but 1 case were judged to be normally distributed on the basis of a clear central peak and symmetrical tails. In 1 experiment (Fig. 11) the normalized data showed a distinct negative skew after training; consequently, nonparametric statistics were used, with the median used to 
Figure 2. Responses of the tail, a tail motor neuron, and 2 sensory neurons in the pleural VC cluster during application of the sequence of training shocks to a site on the tail. The sensory neuron that was activated by the shock (the "trained cell") showed a long-lasting slow depolarization following the training sequence, while the unactivated control sensory neuron showed the opposite effect - a slow hyperpolarization. The basal tension in the tail showed a rapid decay phase followed by a much slower decay, with the tension in this case returning to the baseline level in $35 \mathrm{~min}$. In addition, the motor neuron remained depolarized until the next test, 10 min later.

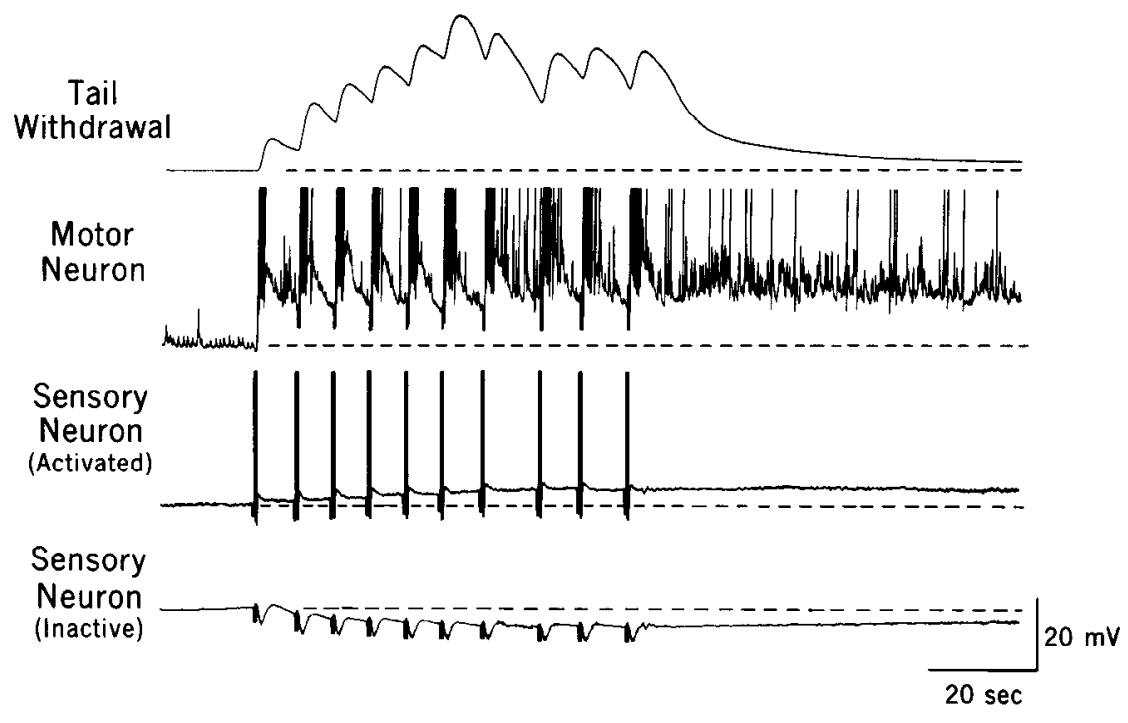

ters and Byrne, 1983b, 1985). The mean ( \pm SEM) peak depolarization of the activated sensory cells $(3.8 \pm 0.6 \mathrm{mV})$ was significantly greater than that of the inactive sensory cells $(-1.4 \pm$ $0.6 \mathrm{mV} ; t_{13}=6.3, p<0.001$ ). This difference in peak depolarization was similar to that observed after a cellular associative conditioning protocol (Walters and Byrne, 1983b) and after a protocol modeled on those used to produce long-term potentiation (LTP; Walters and Byrne, 1985).

In every case the training sequence caused massive excitatory input to the monitored tail motor neuron (Fig. 2). The motor neuron then usually remained depolarized for at least $10 \mathrm{~min}$ afterwards, until the first test after training, at which point it was briefly hyperpolarized back to its original level by intracellular current injection through the recording electrode or through a second intracellular electrode. This hyperpolarization of the motor neuron to a constant level for each test eliminated the possible contribution of anomalous rectification in the motor
Figure 3. Responses to test stimulation of the trained site before and after training. $A$, Increase in magnitude of tail-withdrawal response. The test stimulus was applied at the arrow in each case. Responses were measured (with the CNS connected) $20 \mathrm{~min}$ before, $10 \mathrm{~min}$ after, and $110 \mathrm{~min}$ after training. $B$, Increase in magnitude and duration of extracellularly recorded afferent responses in the posterior pedal nerve (Lp9) innervating the tail. The responses were measured (with the CNS disconnected) $10 \mathrm{~min}$ before, $20 \mathrm{~min}$ after, and $120 \mathrm{~min}$ after training. In this case, the test stimulus consisted of two $5 \mathrm{msec}$ pulses separated by $40 \mathrm{msec}$ (shock artifacts visible at beginning of burst). $C$, Increase in amplitude of monosynaptic EPSP from tail sensory ncuron to tail motor neuron measured $10 \mathrm{~min}$ before, $10 \mathrm{~min}$ after, and 120 min after training. On the $10 \mathrm{~min}$ test, the EPSP reached spike threshold in the motor neuron (the spike is clipped by the pen recorder). The bridge artifact in the lowest trace has been reduced to display the sensory cell spike clearly.

\section{BASELINE}

A. Tail Withdrawal

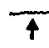

4

B. Afferent Activity
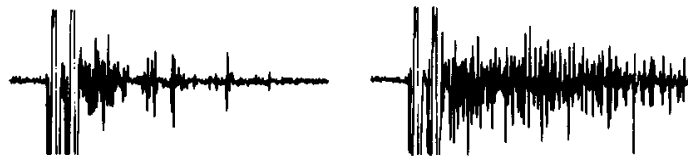

C. Sensory EPSP

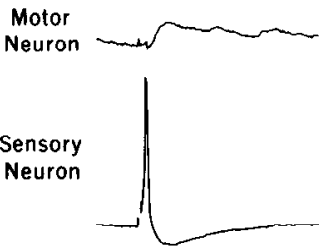

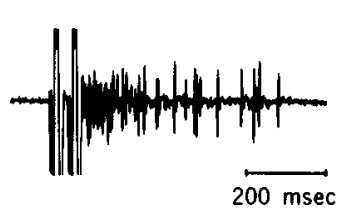

\section{$10-20 \mathrm{MIN}$}
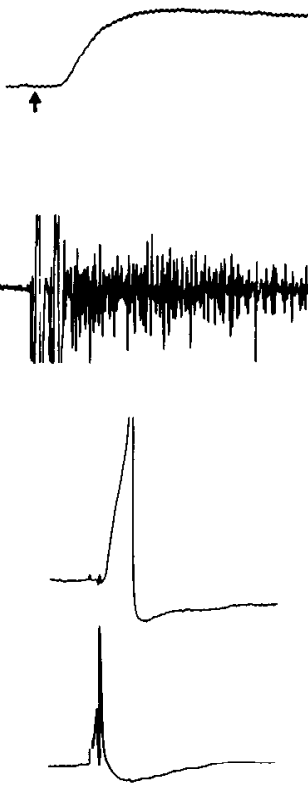

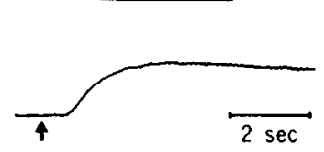

$200 \mathrm{msec}$

2 HOURS

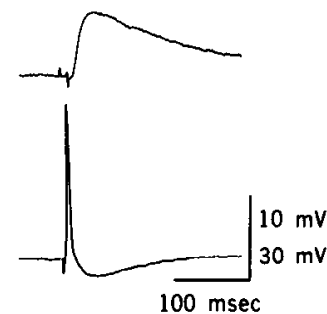


neuron to any EPSP facilitation observed (see below).

Each train of shock caused a tail contraction that was superimposed on the previous contraction, and which was often facilitated compared to the first contraction, leading to a buildup of tension during the sequence. Although most of the tension decreased rapidly (Fig. 2), a residual slowly decaying component then became evident, with return to the baseline level not occurring until $49 \pm 13 \mathrm{~min}$ after training.

\section{Site-specific sensitization of tail-withdrawal test responses}

An example of the tail-withdrawal responses to weak test stimulation applied to the trained site is illustrated in Figure $3 \mathrm{~A}$. Prior to training, the response was very weak, just above threshold for an observable contraction. When tested both 10 and 110 min after training, the amplitude of the response was clearly enhanced. Examination of the pooled data (Fig. $4 A$ ) revealed that, although the untrained site showed transient cnhancement, the trained site displayed significantly greater enhancement, both 10-20 min and 110-120 min (see legend, Fig. 4) after training $\left(t_{6}=2.53\right.$ and 2.15 , respectively, $p<0.05$ in each case). In 3 of 3 animals tested again 3 and $4 \mathrm{hr}$ after training, the trained site remained enhanced relative to the untrained site. In addition to the site-specific enhancement of the amplitude of the tail withdrawals, in every animal there was a clear increase in duration of the tail-withdrawal response elicited from the trained site. The change in duration could not be quantified because the withdrawals were often longer than the test intervals following the training.

In these experiments tests were conducted alternately with the tail connected to the CNS and reversibly disconnected from the CNS by blocking axonal conduction in the posterior pedal nerve (Fig. 1). This allowed monitoring of the peripheral effects of training on the tail-withdrawal reflex (Fig. 4B). The peripheral responses showed suggestive evidence of short-term site-specific sensitization on the 10-20 min test (trained vs untrained site, $t_{6}=1.74, p<0.10$ ) but no site-specific sensitization persisting on the 110-120 min test (trained vs untrained site, $t_{6}=1.33$ ). Short-term peripheral site-specific sensitization has also been observed in deganglionated preparations, lacking any CNS (unpublished observations). Although the periphery alone can show site-specific sensitization, an important central contribution is indicated by the observation that, on both the 10-20 and the 110-120 min tests, the tail-withdrawal responses to testing the trained site were significantly greater with the CNS connected to the tail than when it was disconnected $\left(t_{6}=2.10\right.$ and 2.01 , respectively, $p<0.05$ in each case; Fig. 5). There was little difference in responses to the untrained site with and without the CNS attached to the tail (Fig. 5, Control Site), suggesting that central changes are not essential for expression of the general sensitization seen in this preparation after training.

\section{Site-specific enhancement of afferent activity}

Disconnecting the tail from the CNS on alternate tests (Fig. 1) also allowed measurement of afferent activity in the posterior pedal nerve in the absence of efferent activity. An example of afferent activity elicited by the test stimulus at the trained site is illustrated in Figure $3 B$. There was a clear increase in the number of extracellular spikes and in the duration of the burst elicited by the test stimulus both 20 and 120 min after training. In the 0.5 sec period in which spikes were counted, the mean frequencies on (1) the last baseline trial, (2) the 10-20 min test,
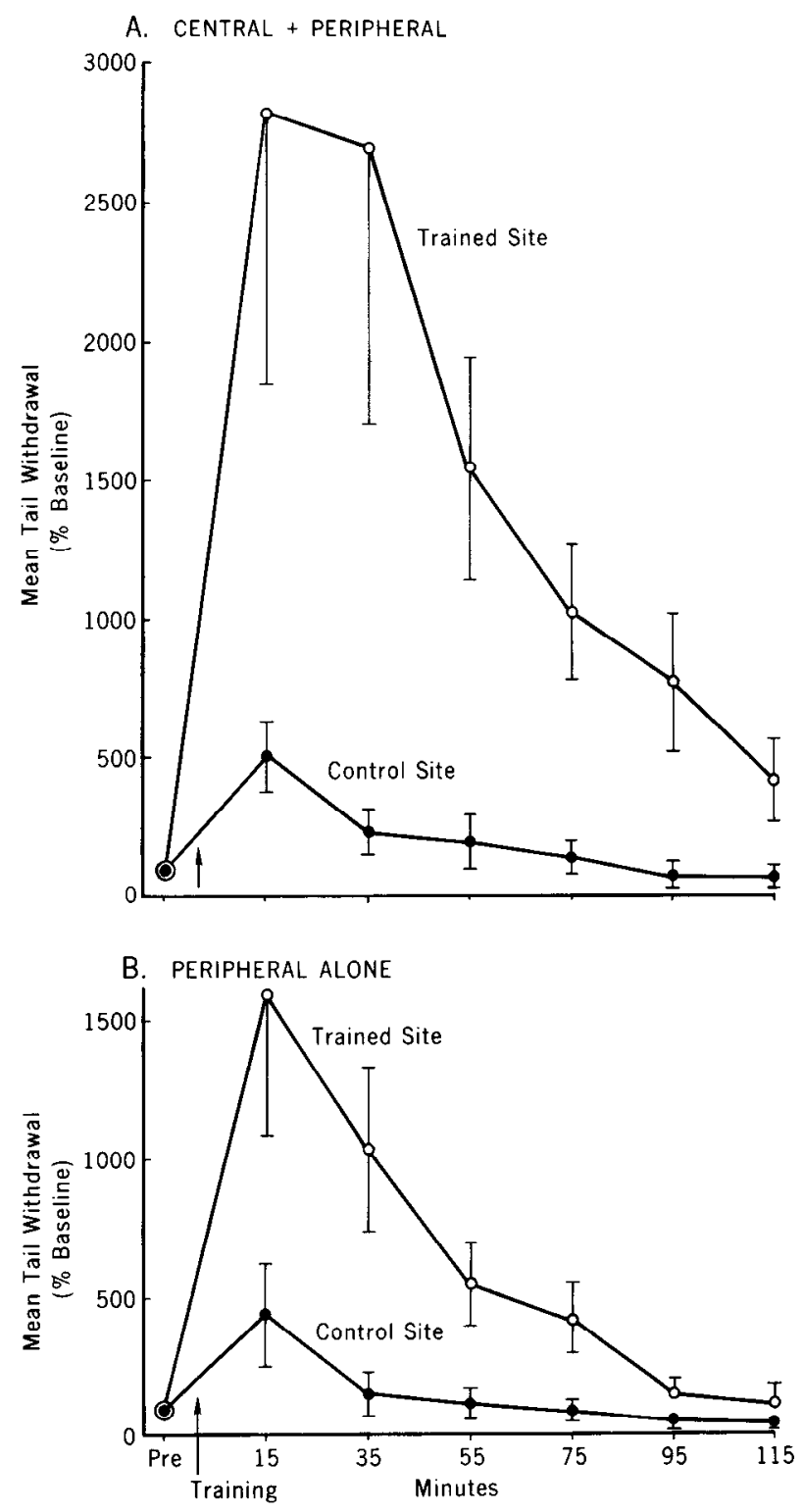

Figure 4. Site-specific sensitization of tail-withdrawal responses. The training sequence was delivered at the arrow. Because alternate testing trials were run with the CNS connected and disconnected, and the order varied in different animals, each point represents a combination of trials that had been run either $5 \mathrm{~min}$ before or $5 \mathrm{~min}$ after the indicated time (e.g., the $15 \mathrm{~min}$ point contains data collected 10 and $20 \mathrm{~min}$ after training). $A$, Change in responses mediated by the combined central and peripheral components of the tail-withdrawal reflex (CNS connected). $B$, Change in responses mediated by the peripheral component alone (CNS disconnected). The CNS was reversibly disconnected by preventing conduction in nerve $\mathrm{Lp} 9$ with $\mathrm{MgCl}_{2}$ (see Fig. 1). The error bars in this and the other figures represent the SEM.

and (3) the 110-120 test were, respectively, for the trained site 41,75 , and $51 \mathrm{~Hz}$, and for the control sitc 42,46 , and $35 \mathrm{~Hz}$. Analysis of the group data (Fig. 6) indicated that the afferent response to testing the trained site was significantly greater than the response to the untrained site on both the 10-20 and the $110-120$ min tests $\left(t_{6}=2.33\right.$ and 2.04 , respectively; $p<0.05$ in each case). There was a low rate of spontaneous background activity from the periphery prior to training (mean rate $=2.0 \pm$ $1.4 \mathrm{~Hz}$ ). After training, afferent background activity increased to $5.7 \pm 2.4 \mathrm{~Hz}$ on the $10-20 \mathrm{~min}$ test and to $3.0 \pm 1.6 \mathrm{~Hz}$ on 


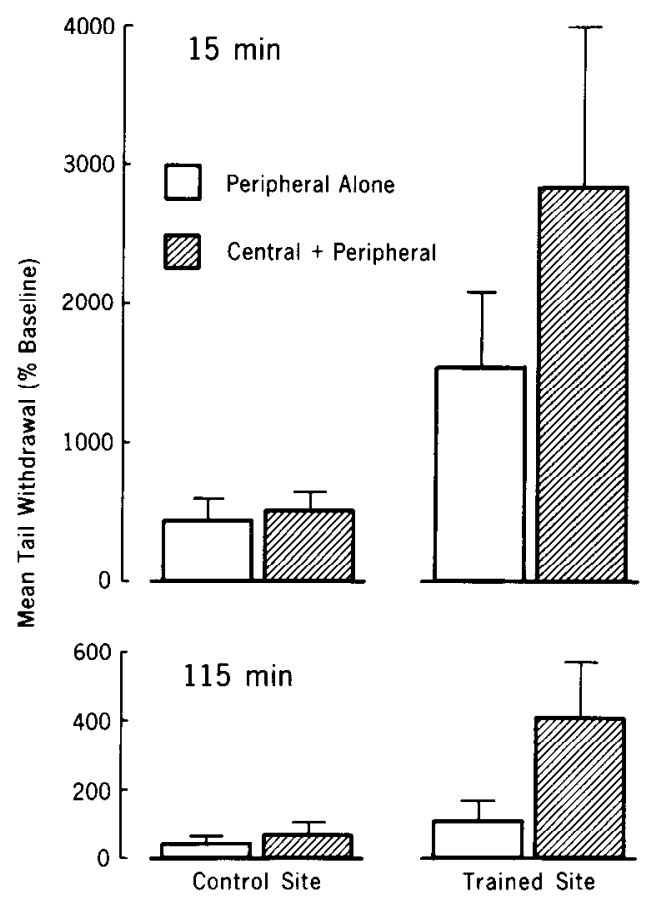

Figure 5. Significant contribution of a central component to site-specific sensitization of tail-withdrawal reflex. $15 \mathrm{~min}$ refers to the 10 and $20 \mathrm{~min}$ tests, $115 \mathrm{~min}$ to the 110 and $120 \mathrm{~min}$ tests. The control site, expressing only general sensitization, showed no significant difference in withdrawal amplitude when tested 15 or $115 \mathrm{~min}$ after the training sequence. The trained site, expressing site-specific sensitization, showed significantly larger responses when tested with the CNS connected to the tail (central + peripheral) than when it was reversibly disconnected (peripheral alone), both 15 and 115 min after training.

the 110-120 min test. This background afferent activity was quite small compared to the activity evoked by the test pulses.

\section{Site-specific enhancement of sensory neuron EPSPS}

An example of the EPSPs (measurcd in a tail motor ncuron) from a sensory neuron that was activated by the training stimulus is illustrated in Figure 3. Following the training sequence the monosynaptic EPSP from this cell showed dramatic facilitation compared to its baseline level. Analysis of the group data (Fig. 7) showed that the EPSPs from "trained" sensory neurons (activated by the cutaneous training sequence) were significantly enhanced compared to EPSPs from nearby unactivated control sensory neurons, both 10 and $120 \mathrm{~min}$ after training $\left(t_{7}=2.64\right.$ and $3.59, p<0.025$ and 0.005 , respectively). The control sensory neurons, however, showed significant facilitation compared to baseline at $10 \mathrm{~min}\left(t_{7}=3.64, p<0.005\right)$ but not at $120 \mathrm{~min}$ $\left(t_{7}=-0.65\right)$, indicating that, in the CNS, the training sequence produced transient general heterosynaptic facilitation, as well as the more powerful and longer-lasting site-specific enhancement of the trained cells. Prior to training, the EPSPs of the trained and control cells were nearly identical $(5.3 \pm 1.7$ and $5.5 \pm 1.4 \mathrm{mV}$, respectively). In neither the trained nor the control sensory neurons were significant differences in EPSP magnitude observed between the trials when the CNS was connected and the trials when the CNS was disconnected, demonstrating that these central neuronal correlates of site-specific sensitization and of general sensitization do not require constant input from the periphery. In addition to firing action potentials during the $45 \mathrm{sec}$ training sequence, there was another difference

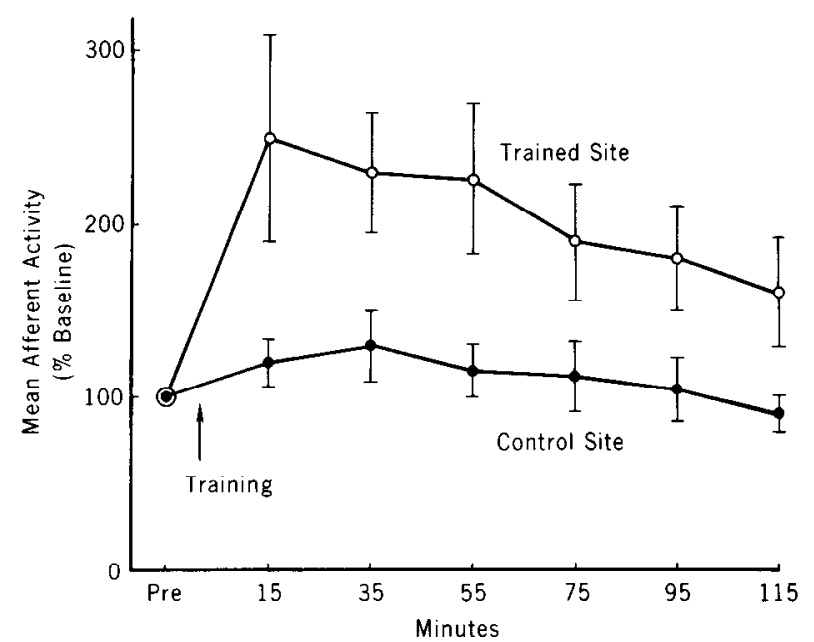

Figure 6. Site-specific sensitization of afferent nerve activity. The extracellularly recorded responses to the test stimuli (with the CNS reversibly disconnected from the tail) showed significantly greater enhancement at the trained site than at the control site. Afferent activity represents the number of spikes observed within $0.5 \mathrm{sec}$ of peripheral test stimulation and is expressed as a percentage of the baseline number [a mean of 20.5 spikes $(41 \mathrm{~Hz}$ ) for the site to be trained and 21 spikes $(42 \mathrm{~Hz})$ for the control site]. Background activity was negligible (see text).

between the trained and control sensory neurons that had to be excluded as a possible cause of the differences in EPSP magnitude: During each test trial the trained sensory neurons, but not the control sensory neurons, were activated a second time by the cutaneous test pulse used to measure tail withdrawal and afferent activity (see Materials and Methods). The potential contribution of these extra test spikes to the EPSP enhancement was ruled out by 2 observations. First, in a separate study in which no cutaneous test stimuli were delivered (see also results on action potential threshold below), and thus no extra test spikes were produced, the trained sensory neurons still showed dramatic facilitation $(592 \pm 93 \%$ at $10 \mathrm{~min}$ and $238 \pm 50 \%$ at $120 \mathrm{~min}$ ), and the responses were significantly enhanced compared to the control sensory neuron EPSPs $\left(t_{s}=2.80\right.$ and 3.45, respectively; $p<0.025$ and 0.01 ). Second, during the baseline period in the study of Figure 7, when the cells that would later be trained were being activated twice in each test while the control cells were activated only once, there was no difference in EPSP magnitude between the 2 sets of cells. Specifically, different preparations received from 2 to 4 baseline trials. Expressing the response on the last baseline trial as a percentage of the response on the first baseline trial, the EPSPs from cutaneously activated sensory neurons (having extra spikes) declined to $64 \pm 7 \%$ and the control sensory neurons' EPSPs to $68 \pm 6 \%$. Therefore, the difference in EPSP magnitude between the trained and control cells is due to the high-frequency activation of the trained cells during the training sequence and not to differences in spike number during the testing.

\section{Site-specific reduction of action potential threshold in sensory neurons}

Walters and Byrne (1985) found a relative reduction in action potential threshold of tail sensory neurons activated by a training sequence applied to the nerve innervating the tail in an isolated ganglia preparation. Because this result was found after application of a sequence analogous to that applied to the tail 


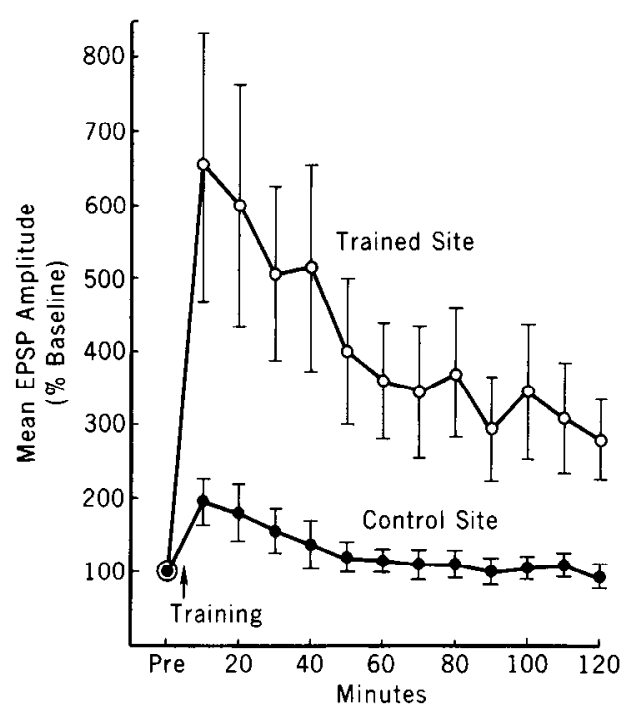

Figure 7. Site-specific enhancement of sensory neuron EPSPs. The intracellularly recorded monosynaptic EPSPs from tail sensory neurons innervating the trained site (activated by the training sequence) showed significantly greater enhancement than control sensory neurons that were not activated by stimulation of the trained site. All EPSPs were measured concurrently in a single tail motor neuron in each preparation. After training the motor neuron was brought to approximately the same membrane potential on each test as observed during the baseline tests by passing hyperpolarizing current into the soma.

in the present investigations, the sensory neurons whose changes in EPSP amplitude are displayed in Figure 7 were examined to see if similar relative changes occurred in threshold. Each sensory neuron was tested with a rapid, ascending series of intracellular depolarizing pulses injected into the cell soma in order to determine the soma action potential threshold (as well as the amplitude of the monosynaptic EPSP to a tail motor neuron; Fig. 7). On both the 10 and 120 min tests action potential threshold was reduced in sensory neurons activated by the training sequence compared to control sensory neurons, but these differences were not significant. To see if significant differences might be revealed with a larger sample size, the data were pooled from (1) the studies in which behavioral responses were measured concurrently with electrophysiological responses (Figs. 37) and (2) the study mentioned above in which EPSPs were measured in the absence of behavioral testing. The training and intracellular testing protocols were identical in each study. Figure 8 shows the pooled results. The trained cells showed significantly lower action potential thresholds at both $10 \mathrm{~min}\left(t_{13}=\right.$ $2.64, p<0.025)$ and $120 \mathrm{~min}\left(t_{10}=2.00, p<0.05\right)$. Thus, sitespecific sensitization is associated with short- and intermediateterm enhancement of the excitability of the sensory neuron soma.

\section{Regenerative bursts and EPSP enhancement correlated with long-term reflex enhancement}

The vigor of the tail-withdrawal responses in the semi-intact preparation begins to decline after several hours, presumably because of fatigue and possibly because of degenerative changes. To begin examining longer-term correlates of site-specific sensitization while avoiding the problems of fatigue and degeneration, the intact animal was trained on 1 day and tested again the next day both behaviorally (intact) and electrophysiologically (semi-intact). The training procedure was changed so that, instead of a very restricted site, an extensive region on 1 side

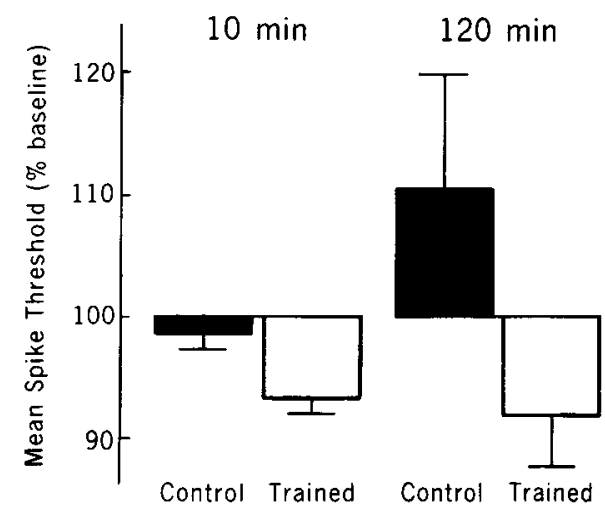

Figure 8. Site-specific enhancement of sensory cell excitability. Threshold for action potential generation in the soma was determined with a series of progressively increasing $10 \mathrm{msec}$ intracellular depolarizing pulses. Trained cells (activated by the tail shock) showed significant reduction in action potential threshold compared to untrained control sensory cells 10 and $120 \mathrm{~min}$ after training.

of the animal was stimulated (see Materials and Methods). This was done for 3 reasons. First, with approximately 200 sensory neurons in each VC cluster (Walters et al., 1983a), it takes time to find the few VC cells innervating any given point on the animal, and, moreover, the cutaneous test stimuli involved in such a search can themselves cause complicating sensitization (Walters et al., 1983b). Second, in 2 preparations examined in the above study the trained cell began to exhibit regenerative bursting responses (Walters et al., 1983b) after training, while none of the control cells showed bursting responses. A training procedure that activated many sensory neurons would increase the chances of detecting significant changes in the fraction of cells that burst regeneratively as a consequence of being activated during training. Third, biochemical investigations of sensory neuron plasticity (e.g., Ocorr et al., 1985) are made easier if analysis can be made of the entire VC cluster, rather than individual cells in this sensory cluster. It has not been shown, however, that significant behavioral and electrophysiological modifiability is induced by massive stimulation of a large part of the sensory cluster. The strong training shocks applied over half of the dorsal surface of the tail, the ipsilateral midbody, and the outer surface of the ipsilateral parapodium should activate up to a third of the sensory neurons in the ipsilateral VC cluster, and none in the contralateral cluster since the stimuli were not delivered near the midline (Walters et al., 1983a, and unpublished observations). Within the tail and parapodial regions of the cluster - the regions that I sampled (see Materials and Methods)-an even larger fraction of the cells should be activated.

In 5 of 7 animals subsequently tested electrophysiologically, behavioral measurements were made in the intact animal 20$24 \mathrm{hr}$ after the spatially extensive training sequence, using procedures described in the preceding paper (Walters, 1987). Tailwithdrawal responses evoked by the weak electrical test stimulus applied to the trained side of the tail appeared stronger than the responses to stimulation of the control side in 4 of 5 animals, but this difference was not statistically significant using the crude subjective measure described (Walters, 1987). The duration of the siphon withdrawals, which can be measured more accurately by eye than can tail withdrawal, showed a clear enhancement in response to test stimulation of the trained side compared to stimulation of the control side (trained: $136 \pm 45 \mathrm{sec}$; control: 
Figure 9. Regenerative bursting response associated with site-specific sensitization. VC sensory neurons and tail motor neurons were recorded $1 \mathrm{~d}$ after application of an electrical training sequence to 1 side of the animal. $A$, Typical response of a "control" sensory neuron on the untrained side. The test action potential was elicited by a 10 $\mathrm{msec}$ depolarizing pulse injected into the sensory neuron soma. $B$, Example of a "trained" sensory neuron's regenerative bursting response to the same brief test depolarization as applied in part $A$. This sensory neuron's receptive field (near the tail) was within the extensive ipsilateral area given the training sequence.
A. Control side

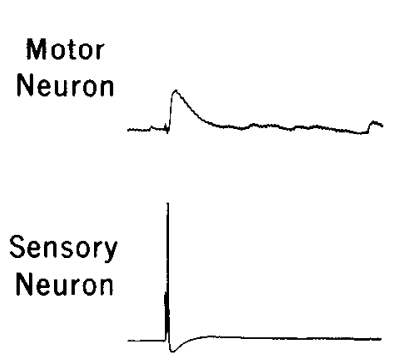

B. Trained side

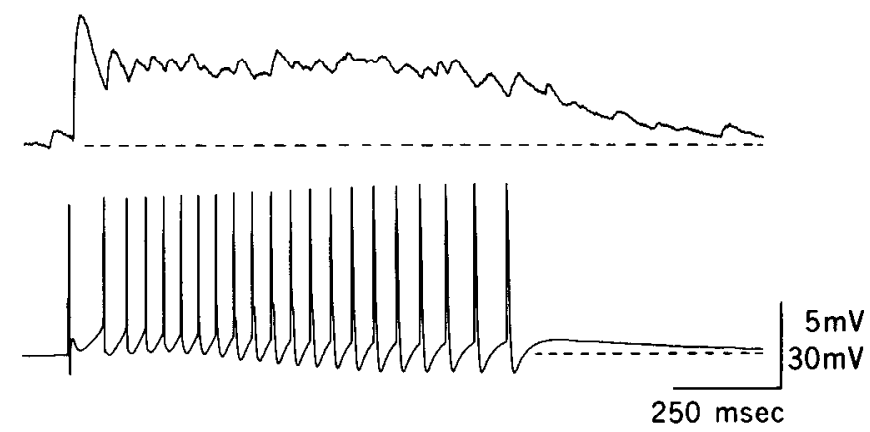

$38 \pm 7 \mathrm{sec} ; t_{4}=2.47, p<0.05$ ), indicting that spatially extensive training stimulation can result in site-specific sensitization that lasts at least $1 \mathrm{~d}$. Although the observer was "blind" to the purpose of the experiment and to the training history of the animals, it is possible that differences in posture or tonus on the trained side might have influenced the measurements. No obvious lesions were observed on the trained side.

Each animal was then surgically reduced to the semi-intact tail preparation (Fig. 1) under $\mathrm{MgCl}_{2}$ anesthesia and allowed to equilibrate for an hour following washout of the $\mathrm{MgCl}_{2}$ with artificial seawater. After homologous tail motor neurons in each pedal ganglion were identified, the sensory neuron sampling procedure was applied to each pleural VC cluster in turn. The choice of side to start with was alternated in successive preparations, and each experiment was conducted "blind."

Figure 9 illustrates the 2 cellular differences observed between the trained and control sides of the animal: (1) training caused some of the ipsilateral sensory neurons to respond to the brief, $10 \mathrm{msec}$, intracellular depolarizing test pulse with a long-lasting, regenerative burst of spikes, and (2) training resulted in larger EPSPs from ipsilateral tail sensory to tail motor neurons. Analysis of the group data revealed that the fraction of sensory neurons that fired regenerative bursts to the intracellular test stimulus was significantly greater on the trained side than the control side (Fig. 10A; $t_{6}=3.74, p<0.005$ ). On the trained side, single bursts contained from 2 to 35 spikes (the longest lasted $2.4 \mathrm{sec}$ ), with a mean burst of $8.1 \pm 6.0$ spikes, and 6 of 7 animals had at least 1 bursting cell. On the control side, 1 animal exhibited 1 bursting cell, and this burst contained only 2 spikes. In 5 of the animals homologous motor neurons were identified in each pedal ganglion and held throughout the sensory neuron sampling procedure. In each case at least 3 sensory neurons on each side were found to evoke measurable monosynaptic EPSPs in the motor neuron. Therefore, the mean amplitude of the 3 largest sensory EPSPs in the motor neuron in each side was compared to the mean on the contralateral side. The mean EPSP amplitude was significantly larger on the trained side than on the untraincd side (Fig. $10 B ; t_{4}=2.63, p<0.05$ ). Although in 4 of 5 preparations a larger fraction of the sensory neurons on the trained side had connections to the motor neuron than did the sensory neurons on the untrained side, this difference was not significant, and the mean fraction was the same on each side: $49 \%$. A larger sample will be necessary to evaluate the possibility that training increases the number of detectable connections from the sensory neurons to the tail motor neurons (cf. Carew et al., 1979).

\section{Site-specific EPSP enhancement occurs in a US pathway during associative conditioning}

If site-specific sensitization is a general phenomenon in Aplysia, it should occur in the unconditioned stimulus (US) pathway during aversive associative learning (see Walters, 1987). A number of studies have now used similar patterns of tail stimulation as the US for associative conditioning in Aplysia (e.g., Carew et al., 1981, 1983; Hawkins et al., 1983; Walters and Byrne, 1983a). In order to test this prediction, unpublished data collected during the associative conditioning study reported by Walters and Byrne (1983a) were analyzed. Although the purpose of this previous study was to analyze the effects of pairing artificial intracellular activation (the CS) with sensitizing neuromodulation caused by a tail shock (the US) delivered outside of the receptive fields of the sensory neurons, in 7 preparations sensory neurons were also recorded that were directly activated by the tail shock US. Thus, these cells were in the US pathway and would be expected to show site-specific EPSP enhancement underlying site-specific sensitization of the US site. Because of a negative skew in the response distribution, these cells were analyzed with nonparametric methods. This skew appeared to be due to the much smaller facilitation in cells whose EPSPs were large to begin with, presumably because of a ceiling effect (baseline EPSPs ranged from 1.5 to $22 \mathrm{mV}$ ). Similar to the pattern observed with the cells activated by the intracellular CS (Walters and Byrne, 1983a), the cells activated by the US showed a gradual increase in their EPSP amplitudes during conditioning, followed by a slow decline after conditioning (Fig. 11). Wilcoxon's signedrank test revealed that the trained (US) cells had significantly greater EPSPs than the control cells (which expressed the effects of general sensitization) both 5 and $30 \mathrm{~min}$ after the fifth (last) training trial ( $n=7, p<0.02$ in each case, 2-tailed). In addition, this site-specific enhancement of EPSP amplitude in the US pathway was very similar to the associative CS enhancement of EPSP amplitude previously reported from some of the same animals (Walters and Byrnc, 1983a).

\section{Activation alone does not explain site-specific EPSP enhancement}

Although the relative enhancement of EPSPs in the CS and US pathways compared to that in unactivated sensory neurons (mediating only the effects of general sensitization) suggests that ADEM is a mechanism underlying long-lasting EPSP enhancement in the semi-intact tail preparation, the possibility re- 

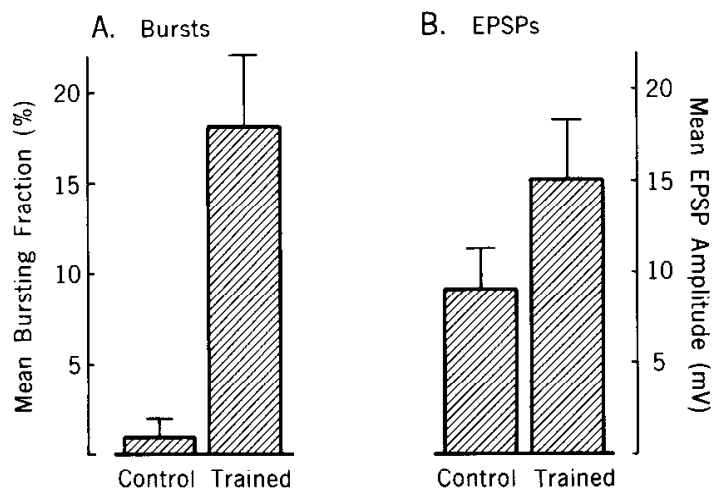

Figure 10. Neuronal correlates of long-term (1 d), site-specific sensitization. Training stimulation was applied to an extensive region encompassing the middle and posterior dorsal surface on half of the intact animal. Electrophysiological testing was conducted a day later in the semi-intact tail preparation. $A$, Regenerative bursting responses in VC sensory neurons. The bursting fraction is the percentage of cells sampled on the trained or control side that responded with a regenerative burst of 2 or more action potentials. $B$, EPSPs from VC sensory neurons. The mean EPSP amplitude of the control and trained sides was the mean of individual means of the 3 largest EPSP amplitudes sampled from each side of each animal.

mained that sensory neuron activation alone might be sufficient to explain the site-specific and temporally specific effects, particularly since posttetanic potentiation has been described in Aplysia mechanosensory neurons (Walters and Byrne, 1984). This possibility was ruled out by an experiment in which sensory neurons were artificially activated with the same pattern of activity observed in the cells activated by the CS and US, except that tail shock was omitted, thus omitting the sensitizing neuromodulation (see Walters and Byrne, 1985, for a similar experiment in the isolated ganglia preparation). Although shortterm posttetanic potentiation (cf. Walters and Byrne, 1984) was observed on the test $5 \mathrm{~min}$ after the last training trial (the mean EPSP in the activated cells was $128 \pm 12 \%$ of the EPSP amplitude in the unactivated control cells, $t_{4}=2.27, p<0.05$ ), at 30 min there was no EPSP enhancement and no difference between the activated and control sensory neurons $\left(t_{4}=0.3\right)$. Thus, enhancement that lasts $30 \mathrm{~min}$ after training in the semi-intact tail preparation requires both activation and sensitizing neuromodulation of the mechanosensory neurons.

\section{Discussion}

Although noxious stimulation has played an important role in many invertebrate models of behavioral and neuronal modifiability, little is known about nociceptive information processing in invertebrates (the leech being a notable exception, e.g., Nicholls and Baylor, 1968). Part of the problem has been a lack of identified nociceptive afferents. Recently, a nociceptive function was indicated for the $\mathrm{VC}$ mechanoafferent neurons innervating the tail, parapodia, and much of the foot and body wall of Aplysia (Walters et al., 1983a). Although these cells respond weakly to light pressure, they show increasing discharges to progressively increasing pressure, with the maximal responses occurring to stimuli that cause tissue damage. Thus, they can be classified as nociceptors (Sherrington, 1906; Burgess and Perl, 1973) with a wide dynamic range (Mendell, 1966). Because of their response properties, restricted excitatory receptive fields, and large "inhibitory" receptive fields, it has been suggested (Walters et al., 1983a) that these mechanoafferents in Aplysia

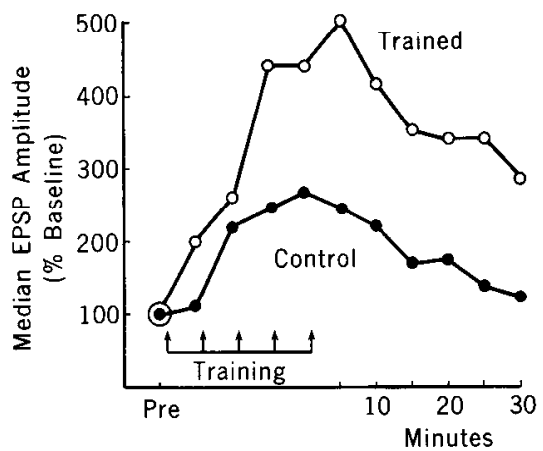

Figure 11. Site-specific EPSP enhancement in a US pathway. Five training trials (arrows) were delivered at 5 min intervals. On each trial a strong $200 \mathrm{msec}$ AC shock was applicd through an implantcd clcctrode in the tail. Four min after each training trial both the sensory neuron innervating the trained site (activated by the training stimulus) and the unactivated control sensory neuron were tested with $20 \mathrm{msec}$ depolarizing pulses injected into the soma. The resulting EPSPs were measured in a common tail motor neuron. Testing continued for $30 \mathrm{~min}$ after training. Results are expressed as medians rather than means because of a negative skew in the response distribution.

combine within individual cells many of the properties that are distributed in mammalian nociceptive systems across primary sensory neurons and wide-dynamic-range sensory interneurons. The present study has extended the functional similarities between nociceptive systems in Aplysia and in other animals by showing that site-specific sensitization of a withdrawal response by noxious stimulation is associated with long-lasting peripheral and central changes that, at least formally, parallel changes that have been described in mammals.

By monitoring the behavior of the tail with a tension transducer in the semi-intact preparation, a more detailed view of the behavioral changes associated with site-specific sensitization was obtained than was possible by visual inspection in the intact animal (Walters, 1987). The strong training stimulus in the semiintact preparation caused a long-lasting increase in basal tension of the tail which might be analogous to the persistent flexion that is often produced in a vertebrate limb by injury (Woolf, 1984). Similarly, intact Aplysia show a long-lasting local withdrawal at an injured site that probably serves several defensive and repair functions (Walters and Erickson, 1986). A peripherally released humoral factor that can act directly on muscle (Cooper and Walters, 1986) may cause part of this persistent withdrawal. During the brief training sequence, successive trains of shock often caused progressively larger withdrawal responses (Fig. 2). This phenomenon was also seen, with longer interstimulus intervals, in a previous study of the tail-withdrawal reflex (Walters et al., 1983b), and formally resembles both the "windup effect" of repeated mechanical stimulation on hindlimb flexion responses in the rat (Woolf, 1984) and the "incremental sensitization" of reflexes discussed by Razran (1971).

While psychologists use the term "sensitization" to refer to nonassociative enhancement of behavioral responses, somatosensory physiologists use the same term to describe enhancement of sensitivity of peripheral (and sometimes central) sensory pathways. It has long been known that cutaneous injury is associated with sensitization of nearby $\mathrm{C}$ polymodal nociceptors in animals (e.g., Witt and Griffin, 1962; Bessou and Perl, 1969) and in humans (e.g., Torebjork et al., 1984). The enhancement of afferent activity observed in Aplysia parallels the increases in afferent activity seen during sensitization of nociceptors in 
mammals. In addition, the small increase in background activity of the afferents in Aplysia after training has a counterpart in the development of spontaneous activity in mammalian nociceptors that sometimes follows noxious stimulation (Bessou and Perl, 1969). It is not yet known whether the changes in afferent activity in Aplysia involve the identified VC cluster sensory neurons. However, the changes in excitability of these cells measured centrally (discussed below) could, in principle, explain the changes observed in afferent activity if parallel changes occurred in the membrane of the peripheral spike-generating zones or receptor regions of these cells. Although the peripheral processes of the VC sensory neurons are not very accessible for intracellular impalement, making a direct test of this hypothesis difficult, preliminary results indicate that the number of action potentials elicited by peripheral stimulation of a VC sensory neuron can be enhanced by sensitizing stimulation of its receptive field (unpublished observations). This effect was observed in $1 \mathrm{VC}$ sensory neuron in the present study, but a larger sample will be necessary to evaluate its significance for site-spccific scnsitization.

Because Aplysia have a PNS as well as a CNS, and also because of the possibility that muscular performance itself can be enhanced (e.g., Weiss et al., 1978; Tritt and Byrne, 1982; Lloyd et al., 1984; Cooper and Walters, 1986), it is not surprising that the tail isolated from the CNS can exhibit site-specific sensitization (Fig. 4B). The fact that the peripheral enhancement has largely decayed within $2 \mathrm{hr}$ may explain why little or no peripheral memory was seen after analogous training in the intact animal (Walters, 1987), since the latter study did not test until at least $2 \mathrm{hr}$ after training. Even in the short term, however, a significant central component of site-specific sensitization was revealed on both the behavioral (Fig. 5) and neuronal (Fig. 7) levels. The large increase in the magnitude of the EPSPs from the VC sensory neurons to the tail motor neurons would be expected to contribute to the observed enhancement of tail withdrawal, since these sensory neurons make a substantial contribution to the tail-withdrawal reflex (Walters et al., 1983a). The enhancement of EPSPs is not caused by continued peripheral input after training, since repeatedly blocking nerve conduction had no effect on central EPSP amplitude. In addition, the sitespecific enhancement of EPSP amplitude was quite similar to the pathway-specific enhancement of EPSP amplitude produced by an analogous training procedure applied to peripheral nerves in completely isolated ganglia (Walters and Byrne, 1985). Other methods (e.g., quantal analysis) will be required to assess the degree to which the changes in EPSP amplitude are presynaptic, but the lack of generalized changes in the postsynaptic motor neurons together with the changes in membrane properties observed in the presynaptic cell soma argue in favor of at least a partial presynaptic contribution to the site-specific EPSP enhancement. Similar enhancement of sensory neuron EPSPs has been shown to be correlated with long-term general sensitization of the siphon-withdrawal reflex (Frost et al., 1985), suggesting that site-specific sensitization and long-term sensitization may share common mechanisms.

Central changes associated with cutaneous injury, and with primary and secondary hyperalgesia, have also been examined in mammalian preparations. Interestingly, these changes have been observed in wide-dynamic-range sensory interneurons and have been interpreted as an enhancement of cellular excitability (e.g., Kenshalo et al., 1979; McMahon and Wall, 1984), although the underlying mechanisms are unknown. The VC sensory neurons in Aplysia also show an enhancement of central excitability following strong cutaneous stimulation that is expressed as both a lowering of action potential threshold in the cell soma and as a tendency for the cell soma to fire in regenerative bursts in response to brief depolarization. Even though changes in action potential threshold and regenerative bursts have been observed in these cells before, they have not previously been correlated with behavioral modifications (Walters et al., 1983b; Walters and Byrne, 1985). Because the VC sensory neurons appear to receive no fast excitatory synaptic input within the CNS, and because the centrally recorded bursts that are elicited by brief peripheral stimuli can outlast the duration of the peripheral stimulus (E. T. Walters, unpublished observations), it seems likely that 1 function of these changes may be to increase the number of action potentials (and hence the magnitude of the signal to the CNS) elicited by peripheral stimuli. Central bursting responses would be synergistic with any enhancement of peripheral excitability in these neurons.

The enhanced excitability suggests that presynaptic facilitation may be only one of a number of changes in the sensory neuron that increase the cell's signaling effectiveness. Indeed, most of the cell may change, not only the synapses in the pedal ganglion, but also the cell soma in the pleural ganglion and perhaps the receptive regions in the periphery. An interesting possibility is that a single mechanism, a decrease in neuromodulator-sensitive K current, e.g., "S-current" (Pollock et al., 1985) and Ca-activated $\mathrm{K}$ current (Walsh and Byrne, 1985), might contribute to multiple changes in many regions of the cell.

Perhaps the most notable feature of site-specific sensitization in the intact animal (Walters, 1987) is its long duration: Less than a minute of noxious or injurious stimulation can produce behavioral effects lasting at least a week. Site-specific sensitization can thus be considered a robust form of single-trial learning that leads to long-term memory. The present results indicate that, during aversive classical conditioning of Aplysia, this form of learning occurs within the US pathway, both centrally and peripherally, and that the central changes are longer lasting than the peripheral ones. The central changes are, in effect, equivalent to those predicted by Konorski (1967) for cells in the mammalian brain that form the "central representation" of a US. It seems likely that there would be advantages to having a central representation of an injury that could insure protection of the damaged site during healing, even in cases where peripheral mechanisms of sensitization might be compromised by the injury (e.g., by peripheral nerve damage). The cellular mechanisms underlying the central changes produced by injury in Aplysia might be largely the same as those implicated in the CS pathway during cutaneous classical conditioning-ADEM of mechanosensory neurons (Hawkins et al., 1983; Walters and Byrne, 1983a). In the present study this mechanism was indicated by the inability of either sensitizing neuromodulation alone or cellular activation alone to produce long-term enhancement, in contrast to the ability of paired activation and neuromodulation (both components being evoked simultaneously by noxious stimulation of the sensory neuron's receptive field) to cause sitespecific enhancement. A role for this mechanism in site-specific sensitization is also suggested by the similarities in changes of EPSP amplitude and action potential threshold to those observed previously in a systematic analysis of ADEM in the VC sensory neurons in isolated ganglia (Walters and Byrne, 1985). 
Athough biochemical mechanisms underlying general sensitization and cutaneous classical conditioning have been investigated in the short term (e.g., Kandel and Schwartz, 1982; Abrams et al., 1985; Ocorr et al., 1985, 1986), examination of long-term biochemical correlates of learning in Aplysia has just begun (Montarolo et al., 1985). Site-specific sensitization may be a particularly useful paradigm for investigating long-term cellular memory. In addition to being easily and reliably produced, it is long lasting, and the underlying cellular changes can be induced simultaneously in a large fraction of the sensory neurons.

Note added in proof: Recently, Scholz and Byrne (1986) have found similar changes involving sensory neuron soma membrane conductances $24 \mathrm{hr}$ after site-specific training.

\section{References}

Abrams, T. W., L. Eliot, Y. Dudai, and E. R. Kandel (1985) Activation of adenylate cyclase in Aplysia neural tissue by $\mathrm{Ca}^{2+} /$ calmodulin, a candidate for an associative mechanism during conditioning. Soc. Neurosci. Abstr. 11: 797.

Bessou, P., and E. R. Perl (1969) Response of cutaneous sensory units with unmyelinated fibers to noxious stimuli. J. Neurophysiol. 32: 1025-1043.

Burgess, P. R., and E. R. Perl (1973) Cutaneous mechanoceptors and nociceptors. In Handbook of Sensory Physiology: Somatosensory System, A. Iggo, ed., pp. 29-78, Springer, Heidelberg.

Carew, T. J., V. F. Castellucci, and E. R. Kandel (1979) Sensitization in Aplysia: Restoration of transmission in synapses inactivated by long-term habituation. Science 205: 417-419.

Carew, T. J., E. T. Walters, and E. R. Kandel (1981) Classical conditioning in a simple withdrawal reflex in Aplysia californica. J. Neurosci. 1 : 1426-1437.

Carew, T. J., R. D. Hawkins, and E. R. Kandel (1983) Differential classical conditioning of a defensive withdrawal reflex in Aplysia californica. Science 219: 397-400.

Cooper, B. F., and E. T. Walters (1986) Humoral factor released by noxious stimulation of Aplysia. Fed. Proc. 45: 888.

Frost, W. N., V. F. Castellucci, and E. R. Kandel (1985) Monosynaptic connections made by the sensory neurons of the gill- and siphonwithdrawal reflex in Aplysia participate in the storage of long-term memory for sensitization. Proc. Natl. Acad. Sci. USA 82: 8266-8269.

Hawkins, R. D., T. W. Abrams, T. J. Carew, and E. R. Kandel (1983) A cellular mechanism of classical conditioning in Aplysia: Activitydependent amplification of presynaptic facilitation. Science 219:400404.

Kandel, E. R., and J. H. Schwartz (1982) Molecular biology of learning: Modulation of transmitter release. Science 218: 433-444.

Kenshalo, D. R., Jr., R. B. Leonard, J. M. Chung, and W. D. Willis (1979) Responses of primate spinothalamic neurons to graded and to rcpcatcd noxious heat stimuli. J. Neurophysiol. 42: 1370-1389.

Konorski, J. (1967) Integrative Activity of the Brain: An Interdisciplinary Approach, University of Chicago Press, Chicago.

Lloyd, P. E., I. Kupfermann, and K. R. Weiss (1984) Evidence for parallel actions of a molluscan neuropeptide and serotonin in mediating arousal in Aplysia. Proc. Natl. Acad. Sci. USA 81: 2934-2937.

McMahon, S. B., and P. D. Wall (1984) Receptive fields of rat lamina 1 projection cells move to incorporate a nearby region of injury. Pain 19: 235-247.

Mendell, L. M. (1966) Physiological properties of unmyelinated fiber projection to the spinal cord. Exp. Neurol. 16: 316-332.

Montarolo, P. G., V. F. Castellucci, P. Goelet, E. R. Kandel, and S. Schacher (1985) Long-term facilitation of the monosynaptic connection between sensory neurons and motor neurons of the gill-withdrawal reflex in Aplysia in dissociated cell culture. Soc. Neurosci. Abstr. 11: 795 .
Nicholls, J. G., and D. A. Baylor (1968) Specific modalities and receptive fields of sensory neurons in $\mathrm{cns}$ of the leech. J. Neurophysiol. 31: 740-756.

Ocorr, K. A., E. T. Walters, and J. H. Byrne (1985) Associative conditioning analog selectively increases cAMP levels of tail sensory neurons in Aplysia. Proc. Natl. Acad. Sci. USA 82: 2548-2552.

Ocorr, K. A., M. Tabata, and J. H. Byrne (1986) Stimuli that produce sensitization lead to elevation of cAMP levels in tail sensory neurons of Aplysia. Brain Res. 371: 190-192.

Pollock, J. D., L. Bernier, and J. S. Camardo (1985) Serotonin and cyclic adenosine $3^{\prime}: 5^{\prime}$-monophosphate modulate the $S$ potassium current in tail sensory neurons in the pleural ganglion of Aplysia. J. Neurosci. 5: 1862-1871.

Razran, G. (1971) Mind in Evolution: An East-West Synthesis of Learned Behavior and Cognition, Houghton Miffin, Boston, MA.

Scholz, K. P., and J. H. Byrne (1986) Long-term sensitization in Aplysia: Biophysical correlates entail sensory neurons. Soc. Neurosci. Abstr. 12: 398 .

Sherrington, S. C. (1906) The Integrative Action of the Nervous System, Cambridge University Press, Cambridge, England.

Torebjork, H. E., R. H. LaMotte, and C. J. Robinson (1984) Peripheral neural correlates of magnitude of cutaneous pain and hyperalgesia: Simultaneous recordings in humans of sensory judgements of pain and evoked responses in nociceptors with C-fibers. J. Neurophysiol. 51: 325-339.

Tritt, S. H., and J. H. Byrne (1982) Neurotransmitters producing and modulating opaline gland contraction in Aplysia californica. J. Neurophysiol. 48: 1347-1361.

Walsh, J. H., and J. H. Byrne (1985) Cyclic AMP and calcium sensitivity of the 5-HT response in tail sensory neurons of Aplysia. Soc. Neurosci. Abstr. 11: 789.

Walters, E. T. (1985) Site-specific sensitization in Aplysia. LTP, regenerative bursting, and a possible link in the evolution of learning. Soc. Neurosci. Abstr. 11: 795.

Walters, E. T. (1987) Site-specific sensitization of defensive reflexes in Aplysia: A simple model of long-term hyperalgesia. J. Neurosci. 7: $400-407$.

Walters, E. T., and J. H. Byrne (1983a) Associative conditioning of single sensory neurons suggests a cellular mechanism for learning. Science 219: 405-408.

Walters, E. T., and J. H. Byrne (1983b) Slow depolarization produced by associative conditioning of Aplysia sensory neurons may enhance $\mathrm{Ca}^{2+}$ entry. Brain Kes. 280: 165-168.

Walters, E. T., and J. H. Byrne (1984) Post-tetanic potentiation in Aplysia sensory neurons. Brain Res. 293: 377-380.

Walters, E. T., and J. H. Byrne (1985) Long-term enhancement produced by activity-dependent modulation of Aplysia sensory neurons. J. Neurosci. 5: 662-672.

Walters, E. T., and M. T. Erickson (1986) Directional control and the functional organization of defensive responses in Aplysia. J. Comp. Physiol. 159: 339-351.

Walters, E. T., J. H. Byrne, T. J. Carew, and E. R. Kandel (1983a) Mechanoafferent neurons innervating tail of Aplysia. I. Response properties and synaptic connections. J. Neurophysiol. 50: 1522-1542.

Walters, E. T., J. H. Byrne, T. J. Carew, and E. R. Kandel (1983b) Mechanoafferent neurons innervating tail of Aplysia. II. Modulation by sensitizing stimulation. J. Neurophysiol. 50: 1543-1559.

Weiss, K. R., J. L. Cohen, and I. Kupfermann (1978) Modulatory control of buccal musculature by a serotonergic neuron in Aplysia. J. Neurophysiol. 41: 181-203.

Witt, I., and J. P. Griffin (1962) Afferent cutaneous C-fibre reactivity to repeated thermal stimuli. Nature 194: 776-777.

Woolf, C. J. (1984) Long term alterations in the excitability of the flexion reflex produced by peripheral tissue injury in the chronic decerebrate rat. Pain 18: 325-343. 